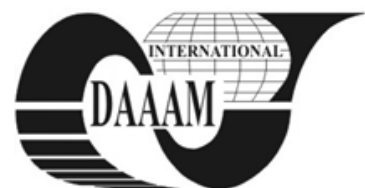

Annals of DAAAM for 2011 \& Proceedings of the 22nd International DAAAM Symposium, Volume 22, No. 1, ISSN 1726-9679 ISBN 978-3-901509-83-4, Editor B. Katalinic, Published by DAAAM International, Vienna, Austria, EU, 2011 Make Harmony between Technology and Nature, and Your Mind will Fly Free as a Bird

\title{
ON THE DETECTION LIMIT OF THE LATERAL BIPOLAR MAGNETOTRANSISTORS
}

\section{PANAIT, C[ornel]; CARUNTU, G[eorge] \& DUMITRASCU, A[na]}

\begin{abstract}
This paperwork presents the structure and the operating conditions of a microsensor realized in the MOS integrated circuits technology based on a bipolar lateral magnetotransistor, where the current deflection effect is dominant. There are established the main noise characteristics of the device and the way of choosing its geometry and material features, which allow the obtaining of high values of signal-tonoise ratio and a high magnetic induction resolution.

Key words: lateral bipolar magnetotransistor, noise current spectral density, shot noise, signal-to-noise ratio
\end{abstract}

\section{INTRODUCTION}

The paper presents the results of research work regarding the analysis and optimization of magnetic microsensor structures realized in MOS integrated circuits technology.

The detection limit is usually defined for conventional Hall devices.

On the basis of adequate models, there have been established the noise main characteristics for bipolar lateral magnetotransistors, where the current deflection effect is dominating.

By using the numerical simulation, the values of the detection limit for different structure devices are compared and it is also emphasized the way in which choosing the geometry and material properties influence on the device performances.

The research should be developed by using new technologies, structures and materials for magnetic microsensors, in order to obtain values for the detection limit as small as possible.

\section{THE GENERAL CHARACTERISTICS}

Figure 1 illustrates the cross section of a magnetotransistor operating on the current deflection principle (Popović,1986).

If the very small magnetic field $\bar{E}_{a}$ is oriented as shown in figure 1 , the electrons are deviated to substrate junction $\left(I_{S}\right)$. Only a few electrons will contribute to collector current $\left(I_{C}\right)$. The area from base region, between the emitter contact and

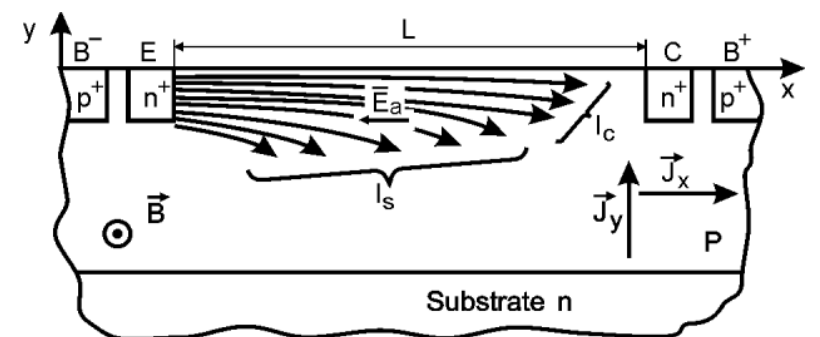

Fig.1. Cross section of lateral magnetotransistor

collector contact, operates as a short Hall plate, and an induction field $\bar{B}_{\perp}$ causes the deflection of current lines. The transverse Hall current will be (Drăgulinescu, 2005):

$$
I_{H}=I_{Y}=(L / Y) I_{C} \mu_{H n} B_{\perp}=\Delta I_{C}
$$

where $\mu_{H n}$ is the Hall mobility of electrons in the $p$-well, and $Y$ is a geometrical parameter given approximately by $y_{j n}<Y<y_{j p}$. Here $y_{j n}$ and $y_{j p}$ denote the junction depths of the collector region and the $p$-well respectively.

A magnetotransistor may be regarded as a modulation transducer that converts the magnetic induction signal into an electric current signal. The supply-current-related sensitivity of the device is defined by:

$$
S_{I}=\frac{1}{I_{C}} \cdot\left|\frac{\Delta I_{C}}{B_{\perp}}\right|=\frac{L}{Y} \cdot\left|\mu_{H n}\right|
$$

\section{SIGNAL-TO NOISE RATIO}

The noise affecting the collector current of a magnetotransistor is shot noise and $1 / f$ noise. Signal-to-noise is defined by (Gray, 1973)

$$
\operatorname{SNR}(f)=\Delta I_{C} \cdot\left[S_{N I} \cdot(f) \cdot \Delta f\right]^{-1 / 2}
$$

where $\Delta f$ denotes a narrow frequency band around the frequency $f$, and $S_{N I}$ is the noise current spectral density. In case of shot noise (1991, Popovic):

$$
S_{N I}=2 q I
$$

where $I$ is the device current.

By substituting (1) and (4) into (3) it is obtained:

$$
\begin{aligned}
S N R(f) & =\frac{1}{\sqrt{2}} \mu \frac{L}{H n} \cdot \frac{I_{C}}{(q \cdot I \cdot \Delta f)^{\frac{1}{2}}} \cdot B_{\perp} \leq \\
& \leq 0.707 \mu_{H n} \frac{L}{Y}\left(\frac{I_{C}}{q \Delta f}\right)^{1 / 2} \cdot B_{\perp}
\end{aligned}
$$

In figure 2 it is shown the $\operatorname{SNR}(f)$ dependence in collector current of three magnetotransistor structures of different materials ( $L / Y=5, \Delta f=1 \mathrm{~Hz}, B=0.2 T$ )

$$
\begin{gathered}
M G T_{1} \text { : Si with } \mu_{H n}=0.15 m^{2} V^{-1} s^{-1} \\
M G T_{2} \text { : Ga Sb with } \mu_{H n}=0.50 m^{2} V^{-1} s^{-1}
\end{gathered}
$$




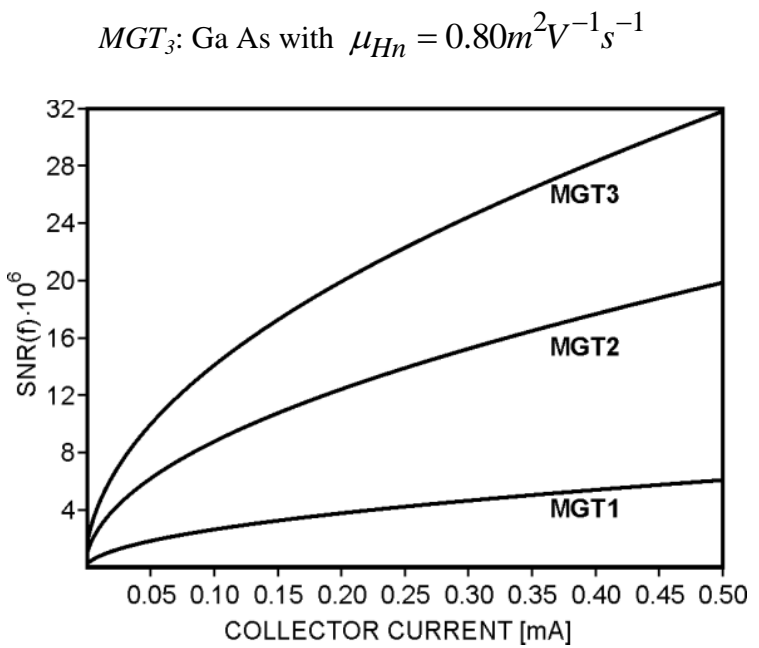

Fig. 2. SNR(f) depending on collector current for three devices of different materials

A high value of carrier mobility causes the increasing of $S N R(f)$. So for $I_{C}=0.2 m A, \operatorname{SNR}(f)$ increases with $60 \%$ for $\mathrm{Ga}$ As comparative with $\mathrm{GaSb}$.

\section{THE DETECTION LIMIT}

A convenient way of describing the noise properties of a sensor is in terms of detection limit, defined as the value of the measured corresponding to a signal-to-noise ratio of one. In case of shot noise, it is obtained from expression (5):

$$
B_{D L} \geq \frac{(2 q \Delta f)^{1 / 2}}{\mu_{H m}} \cdot \frac{Y}{L} \cdot I_{C}^{-1 / 2}
$$

In figure 3 are shown $B_{D L}$ values obtained for three sensors:

$$
\begin{aligned}
& M G T_{1}: \operatorname{Si}\left(\mu_{H n}=0.15 m^{2} V^{-1} s^{-1}\right), \\
& M G T_{2}: \operatorname{GaSb}\left(\mu_{H n}=0.50 m^{2} V^{-1} s^{-1}\right) \\
& M G T_{3} . . \operatorname{GaAs}\left(\mu_{H n}=0.80 m^{2} V^{-1} s^{-1}\right) .
\end{aligned}
$$

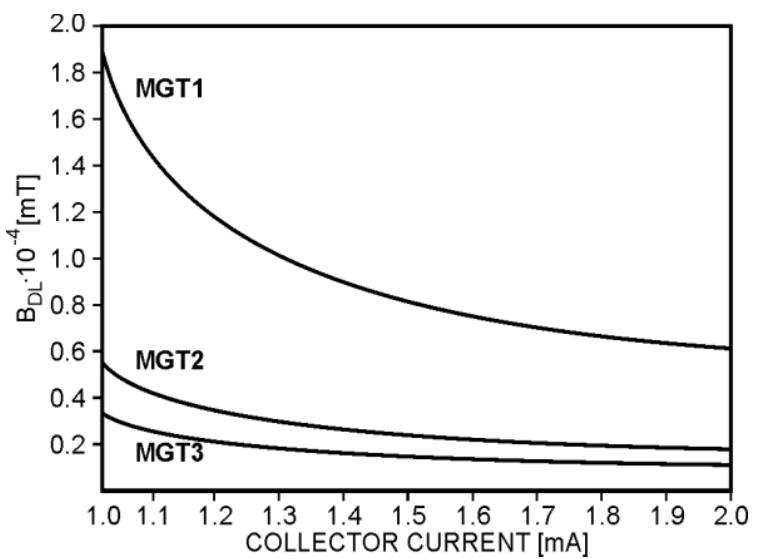

Fig. 3. $B_{D L}$ depending on collector current for three devices of different materials

\section{CONCLUSIONS}

Magnetotransistors have a lower magnetic sensitivity than the conventional Hall devices but they allow very large signalto-noise ratios, resulting a high magnetic induction resolution.

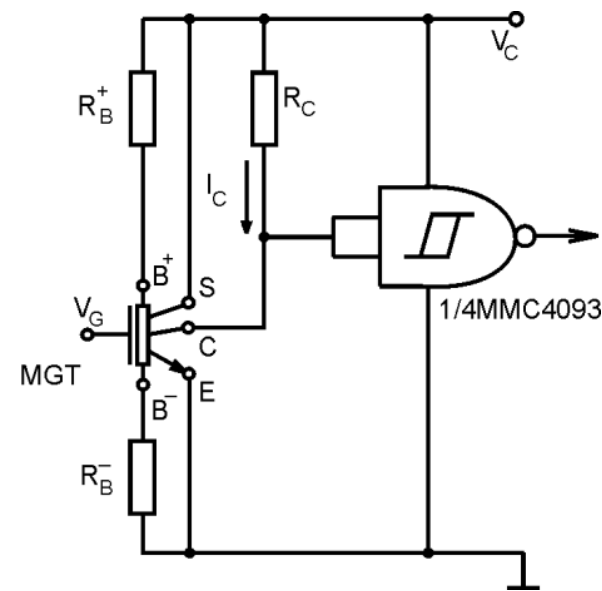

Fig. 4. Transducer with lateral magneto-transistor in CMOS technology

The detection limit $B_{D L}$ decreases under $10^{-5} T$ in case of GaAs at a total collector current of $1 \mathrm{~mA}$.

The analysis of the characteristics of magnetotransistor structures shows that the $W / L=0.5$ ratio is theoretically favorable to high performance regarding signal-to-noise ratio, as well as the offset equivalent magnetic induction.

Also substituting the silicon technology by using other materials such as GaAs or InSb with high carriers mobility values, assures higher characteristics of the sensors.

The transducers with integrated microsensors have a high efficiency and the possibilities of using them can be extended to some measuring systems of thickness, short distance movement, level, pressure, linear and revolution speeds.

Figure 4 shows circuit diagram of a transducer realized with lateral magnetotransistor in CMOS technology (Drăgulinescu 1998).

In the presence of a magnetic field adequately oriented the collector current is very small.

If the magnetic induction decreases, the device current increases which brings about the collector potential variation.

$$
\Delta V_{C}=R_{C} \cdot \Delta I_{C}=R_{C} \frac{L}{Y} \mu_{H n} I_{C} B
$$

\section{ACKNOWLEDGEMENTS}

This research was financially supported by UEFISCDI under the project SIRADMAR no. 12-085 - PN4, "Partnership in priority domain", direction Technologies, Systems and Infrastructures.

\section{REFERENCES}

Drăgulinescu M., „Transducers Realized from Hall Magnetic Microsensors", in the Proceedings of the International Conference, Renewable Sources and Environmental Electro - Technologies, pp. 59-62, Oradea, 27 - 30 May 1998

Drăgulinescu M., "The noise-equivalent magnetic induction spectral density of magnetotransistors", CAS 2005 Proceedings, Sinaia,Romania, Volume 2, pp. 453, 2005.

Gray E.P., C.L. Searle, "Bazele electronicii moderne", Vol I, Editura Tehnica, Bucharest, 1973

***Popovic R.S. (B.E. Jones), "Hall Effect Devices, Magnetic Sensors and Characterization of Semiconductors", Adam Hilger, Bristol, England, 1991

***Popović R.S., R. Widmer, "Magnetotransistor in CMOS technology", IEEE Trans. Electron Devices, 1986, ED-33 1334-40 